\title{
What are the attitudes of health professionals regarding patient reported outcome measures (PROMs) in oncology practice? A mixed-method synthesis of the qualitative evidence
}

Bróna Nic Giolla Easpaig ${ }^{1 *}$ [D, Yvonne Tran¹, Mia Bierbaum¹, Gaston Arnolda1', Geoff P. Delaney ${ }^{2,3,4}$, Winston Liauw ${ }^{5,6}$, Robyn L. Ward ${ }^{7,8}$, lan Olver ${ }^{9}$, David Currow ${ }^{10,11}$, Afaf Girgis ${ }^{3,4}$, Ivana Durcinoska ${ }^{3,4}$ and Jeffrey Braithwaite ${ }^{1}$

\begin{abstract}
Background: The adoption of Patient Reported Outcome Measures (PROMs) in cancer care has been widely advocated, but little is known about the evidence for the implementation of PROMs in practice. Qualitative research captures the perspectives of health professionals as end-users of PROMs and can be used to inform adoption efforts. This paper presents a systematic review and synthesis of qualitative research conducted to address the question: What are the attitudes of health professionals towards PROMs in oncology, including any barriers and facilitators to the adoption of PROMS, reported in qualitative evidence?

Methods: Systematic searches of qualitative evidence were undertaken in four databases and reviewed using the Preferred Reporting Items for Systematic Reviews and Meta-Analyses guidelines. Studies published in English between 1998 and 2018, which reported qualitative findings about the attitudes of health professionals working in oncology towards PROMs were eligible. Studies were assessed using the Critical Appraisal Skills Programme's Qualitative Research Checklist. A sentiment analysis was conducted on primary text to examine the polarity (neutral, positive or negative) of health professionals' views of PROMs. Qualitative meta-synthesis was conducted using a constant comparative analysis.
\end{abstract}

Results: From 1227 articles after duplicates were removed, with 1014 excluded against the screening criteria, 213 full text articles remained and were assessed; 34 studies met the inclusion criteria and were included. The majority of studies were of good quality. Sentiment analysis on primary text demonstrated an overall positive polarity from the expressed opinions of health professionals. The meta-synthesis showed health professionals' attitudes in four domains: identifying patient issues and needs using PROMs; managing and addressing patient issues; the care experience; and the integration of PROMs into clinical practice.

\footnotetext{
* Correspondence: brona.nicgiollaeaspaig@mq.edu.au

${ }^{1}$ Australian Institute of Health Innovation, Macquarie University, North Ryde,

NSW 2109, Australia

Full list of author information is available at the end of the article
}

(c) The Author(s). 2020 Open Access This article is distributed under the terms of the Creative Commons Attribution 4.0 International License (http://creativecommons.org/licenses/by/4.0/), which permits unrestricted use, distribution, and reproduction in any medium, provided you give appropriate credit to the original author(s) and the source, provide a link to the Creative Commons license, and indicate if changes were made. The Creative Commons Public Domain Dedication waiver (http://creativecommons.org/publicdomain/zero/1.0/) applies to the data made available in this article, unless otherwise stated. 
(Continued from previous page)

Conclusions: From the accounts of health professionals, the fit of PROMs with existing practice, how PROMs are valued, capacity to respond to PROMs and the supports in place, formed the key factors which may impede or promote adoption of PROMs in routine practice. To assist policy-makers and services involved in implementing these initiatives, further evidence is required about the relationship between PROMs data collection and corresponding clinical actions.

Trial registration: International Prospective Register of Systematic Reviews (PROSPERO) CRD42019119447, 6th March, 2019.

Keywords: Oncology, Patient reported outcome measures, PROMs, Health professional attitudes, Qualitative synthesis, Implementing practice change

\section{Background}

Promoting patients' engagement with their health care has been viewed as a means of improving the identification of patient needs and priorities and creating opportunities to address those needs during the cancer journey $[1,2]$. Patient-reported outcome measures (PROMs) are derived from patient self-assessment of a variety of health and wellbeing indices, and provide information to health professionals (HPs) about the patient's health status $[3,4]$. PROMs data may relate to one or multiple health-relevant domains including psychological and physical wellbeing, and be collected using a range of electronic and/or paper-based mediums [5]. Studies have identified differences between patient and clinician assessments of outcomes in oncology with regard to treatment side effects, numerous physical symptoms, as well as psychological issues, whereby oncologists only identified a small proportion of the total patients who were experiencing clinical anxiety and depression $[6,7]$. This discordance has not improved over the past two decades $[6,7]$, supporting the need for patients' direct reports. A systematic review found that PROMs may be useful in cancer care, to longitudinally monitor and respond to the impacts of treatments or symptoms on patients' lives [8]. For example, some PROMs are designed to automatically trigger the provision of tailored information to patients to help them address their symptoms and side-effects $[4,9,10]$.

Systematic collection and feedback of PROMs results to the care team is reported to improve processes and outcomes of care $[1,4]$. In a randomised controlled trial, 766 patients receiving outpatient chemotherapy were randomly assigned to an intervention or control group. The control group received standard care with symptoms monitored by the treating clinician while the intervention group, in addition, electronically reported on 12 common symptoms at set times [1]. Patients in the intervention group scored higher on the health-related quality of life measure, showed greater treatment adherence, had fewer hospital admissions and had a higher survival rate at 1 year. Ahmed and colleagues [11] propose that PROMs are useful for comparing treatments and can also be used to evaluate quality improvement activities. Additionally, a systematic review of the effects of PROMs on clinical practice identifies potential benefits in micro-level patient-clinician interactions, predominantly by enhancing communication and revealing psychological and physical issues [5, 12]. However, evidence for the impact of PROMs on clinical practices, such as prompting appropriate referrals, is reported to be weak [5, 13] or ambiguous [8], and a greater understanding of how PROMs may be integrated and used in clinical care has been sought $[3,5]$.

Howell et al. [3] observed that little is known about the evidence concerning the introduction of PROMs into routine practice. A strength of qualitative research is that it captures the perspectives of those involved in interventions or programs, such as during the introduction of PROMs. This information may help to guide the future implementation [14]. Boyce and colleagues [15] undertook a systematic review of qualitative research that examined HPs' views and experiences with PROMs, through which they identified a set of concerns. HPs raised practical concerns about possible increases in workload, especially where PROMs were not fully integrated into existing patient management systems, and highlighted the importance of training. Some HPs were not open to changing their practices and harboured negative attitudes towards PROMs, potentially hindering adoption. In some studies, clinicians suggested that the relative clinical importance of different PROMs needed clarification, and PROMs data needed to be aggregated to contextualise and complement other clinical data. Mixed views were reported about the capacity of PROMs to improve patient care and some clinicians worried about negative impacts on the patientclinician relationship. On the positive side, the Boyce review [15] reported that professionals believe PROMs increased patient education, stimulated better care planning and built confidence in the competence of the professional. 
The Boyce et al. [15] review raised a number of important issues, but excluded qualitative research from mixed-methods studies. Such studies are often used in program development and studies of acceptability and feasibility. While some of the studies examined were in oncology $(n=1)$ and palliative care $(n=5)$, the focus of that review was not disease specific. Extending previous work, the present systematic review and synthesis of qualitative research concerning the attitudes of HPs to PROMs, specific to cancer care, will provide insights to guide implementation efforts.

\section{Review question}

What are the attitudes of HPs towards PROMs in oncology, including any barriers and facilitators to the adoption of PROMs, reported in qualitative evidence?

\section{Methods}

\section{Search strategy}

Search strategies were adopted from Boyce et al. [15], revised to reflect updated terminology and the oncology focus. The strategy contained five blocks of relevant terms and keywords for: 1) patient-reported outcomes, 2) qualitative research, 3) attitudes, 4) HPs, and 5) oncology. Medline, Cinahl, Embase and PsychInfo databases were searched in October 2018, and updated in April 2019 to capture literature published from January 1998 to December 2018. Results of the searches were imported into EndnoteX9 [16] and duplicates removed. Reference lists of included papers were screened. The protocol was registered with Prospero (no. CRD42019119447).

\section{Study inclusion criteria}

Studies were included if they were: 1) qualitative or mixed-method, where the qualitative data was analysed and reported separately; 2) published in English and reported primary findings; and 3) reported attitudes (broadly defined to include views, perceptions and perspectives) of HPs working in oncology towards PROMs. Study selection was documented and is summarised in a Preferred Reporting Items for Systematic Reviews and Meta-Analyses (PRISMA)-compliant flow chart [17](see Fig. 1; Additional file 1).

\section{Study selection}

Four independent reviewers (BNGE, MB, YT, GA) each reviewed a quarter of the titles and abstracts. A random selection of $5 \%$ of title abstracts were jointly reviewed to determine inter-rater reliability. Full text was retrieved for title abstracts which appear to meet inclusion criteria, and the article assessed independently by at least two team members to determine eligibility. Disagreements were resolved by team consensus.

\section{Study quality assessment}

The quality of studies was assessed using the Critical Appraisal Skills Programme, Qualitative Research Checklist [18]. This tool was specifically designed for the assessment of a range of dimensions of quality in qualitative research. This includes items to help assess the "Risk of bias in individual studies" [17] by examining the extent to which researchers considered their potential influence and bias (e.g. "Has the relationship between researcher and participants been adequately considered?"), as well as examining the appropriateness of the methodology and study design in this regard [18].

\section{Data extraction strategy}

A purpose-designed template was used to guide data extraction, which included: citation, year, title, location of study, study aim/s, study setting, study design, data collection method, cancer stream, description of PROMs reported, HP role (where reported), key barriers to PROMs use, key facilitators to PROMs use, key attitudes towards PROMS, and other details noted as important in the study.

\section{Data synthesis and presentation Sentiment analysis}

To assess whether the reported opinions and attitudes of HPs to PROMs were neutral, positive or negative, primary data from results sections were quantitatively textmined and a sentiment analysis conducted. Sentiment analyses use natural language processing to computationally examine the opinions, sentiment and subjectivity in text [19]. Our sentiment analysis was conducted using $R$ versions 3.5.2 and RStudio (v1.1.442) [20, 21]. Sentiment scoring was performed using the Sentimentr package, examining polarity of text by applying existing sentiment dictionaries and taking into account valence shifters in text such as words that act as negators and amplifiers (e.g. I am not very happy) [22]. Words that attracted a sentiment score, such as "patient" or "kind", but which were not used in the context of a positive sentiment were excluded in the sentiment scoring. Polarity scores were generated for each sentence, with 0 representing a neutral sentence, a negative score representing a negative sentiment and a positive score represented a positive sentiment.

\section{Thematic meta-synthesis}

The extracted data for each area of focus (attitudes, barriers and facilitators) were aggregated and thematic meta-synthesis was performed [23, 24]. NVivo 12 


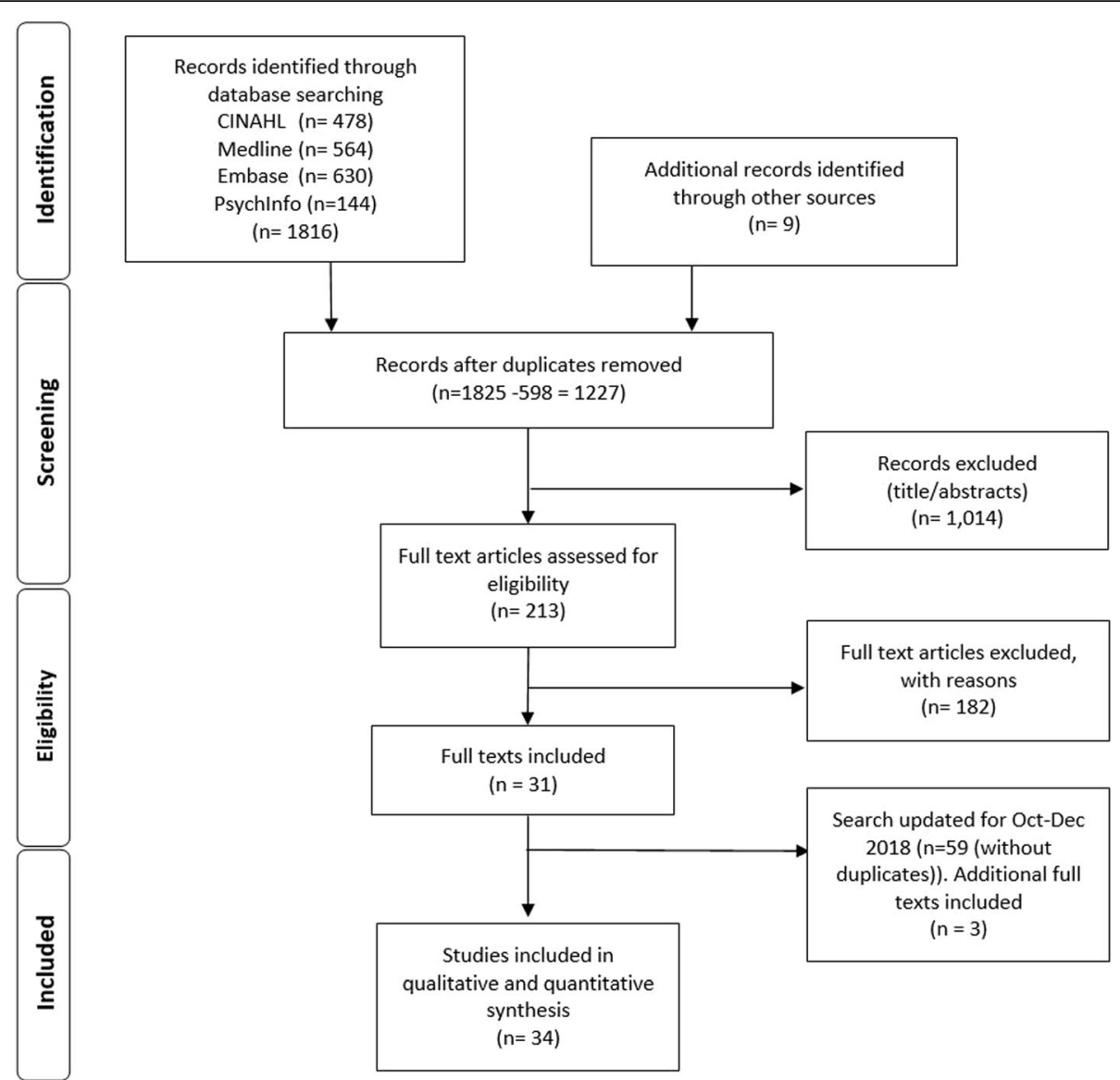

Fig. 1 PRISMA [17] flowchart of search strategy

software was used to manage and assist analysis [25]. In Stage 1, two team members (BNGE, MB) independently undertook line by line coding of subsets of data comprised of study results sections (i.e. including the raw data) and discussion sections (including the authors' interpretations). The review question was sufficiently broad in scope to accommodate an inductive free coding approach. Each member independently developed a codebook on a subset of articles and these were reconciled through discussion to develop a single coding framework. In Stage 2, the framework guided the coding of all data to group codes into descriptive themes. Constant comparative analysis enabled the translation of concepts between studies. The framework was refined as needed during this process and provided a foundation for the development of analytical themes. An iterative process of engaging with the descriptive themes, the review question and raw data was undertaken in Stage 3. This resulted in the development of analytical themes, which while grounded in the data, provide insights and understandings that move beyond the data. Discrepancies were resolved by team discussion.

\section{Results}

Characteristics of included studies

The search conducted for the period January 1998 to October 2018 returned 1218 unique records. The review of the reference lists of included papers identified 9 additional records. The full text of 213 papers was reviewed and 182 papers were excluded on the basis that: 1) qualitative and quantitative findings were not reported separately; 2) it was not possible to distinguish findings from HPs from other groups (e.g. patients); and/or 3) PROMs were not a primary focus of study (i.e., they were captured incidentally). As a result, $31 \mathrm{pa-}$ pers were included. The search conducted for the period October-December 2018 returned 59 unique records which were screened and 56 were excluded for the reasons previously described. This resulted in the inclusion of an additional 3 papers. In total 34 papers were included.

Studies were most commonly conducted in the UK $(n=14)$, the USA $(n=9)$, and Australia $(n=3)$ and included professionals working in a range of settings, typically within clinics or treatment centres $(n=13)$. Ten studies specified the research was undertaken in 
acute care, while some studies were undertaken in a mixture of settings (see Table 1 ).

The majority of studies $(n=24)$ focused on multiple cancer streams. Most study samples included a mixture of HPs, usually a combination of doctors and nurses, while others in addition included managers, administrators and allied health staff. Half of the studies $(n=17)$ used a mixed-methods design. Interviews were usually the sole qualitative method used $(n=22)$. Studies which used multiple qualitative methods paired interviews with focus groups $(n=3)$, with observations, $(\mathrm{n}=1)$ or with a questionnaire $(n=1)$.

In some studies, the focus was on views about PROMs in practice, rather than a specific tool or measure $(n=6)$. The remainder of studies examined a range of PROMs; in which over half of patient reports were collected electronically $(n=18)$. The most common measures were: ESAS $(n=5)$; Problem Checklist $(n=4)$; Distress Thermometer $(n=3)$; and EORTC QLQ-C30 $(\mathrm{n}=3)$ (see Additional file 2 for list of PROMs and abbreviations).

The included studies spanned PROMs development through to implementation; six studies reported on the development of the PROMs tool as well as evaluating it in practice. The majority of studies examined HPs' views in relation to using PROMs in practice, either as part of a pilot or during roll-out $(n=28)$. While all of the studies focused upon HPs' perceptions of PROMs, a number assessed acceptability as a key focus $(n=13)$.

\section{Quality of included studies}

Studies were assessed as meeting between 6 and 10 of the quality criteria, with an average rating of 8.82 . The majority of the studies reviewed $(n=30)$ were rated as satisfying at least 8 quality criteria. The item which received the fewest number of positive ratings was "Has the relationship between researcher and participants been adequately considered?" [18], with only 9 studies considered to have done so (see Table 1).

\section{Sentiment analysis}

From the 34 primary texts, a total of 363 sentences relevant to HPs' attitudes to PROMs were extracted. The mean sentiment score was found to be marginally positive at 0.08 (minimum $=-1.04$, maximum $=1.43$ ), with the highest density of sentences having a neutral sentiment. There was a greater positive density tail, demonstrating a higher number of positive comments. To gauge the meaning and context of the different sentiment scores here are some examples of sentences with a positive, negative and neutral sentiment score:

- "Well the training was excellent, the day that we had at the University was very informative, all the handouts were clear, there was plenty of time as well, and you could, there were loads of opportunities for questions, everything was absolutely splendid in terms of training." This sentence had a positive sentiment score of 1.03.

- "I think of it more in terms of research of standardized questionnaires to evaluate the impact of interventions or peoples experience." This sentence has a neutral sentiment score of 0 .

- "So compared to the system that I'm used to, it seems cumbersome, it adds in too many other things to do to actually get to the people." This sentence has a negative sentiment score of -0.472

Figure 2 shows a density plot for the sentiment scores; there were a greater number of higher scoring positive sentiment sentences than negative ones. Positive comments often occurred when HPs were describing the usefulness of the benefits of the PROM, whereas negative comments were regarding feasibility of implementing PROMs, such as finding it to be time-consuming or cumbersome.

\section{Meta-synthesis}

Four themes were revealed: HPs' attitudes towards identifying patient issues and needs; managing and addressing patient issues; the care experience; and the integration of PROMs into clinical practice (see Fig. 3; Table 2).

\section{Attitudes to identifying patient issues and needs}

This theme captures HPs' attitudes towards the identification of patient issues and needs using PROMs.

Prioritising and focusing on issues PROMs were reported by HPs to be helpful for identifying a wide range of issues implicated in patient wellbeing. This included psychosocial $[10,26,33,51]$, and other quality of life issues which "often are overshadowed by attention to medical aspects of the disease and treatment" [41] (p284)

Where PROMs were completed prior to a consultation, this could aid HP preparation [27, 32, 33, 36] and promote careful reflection on the part of patients [52, 55]. PROMs were also a means to structure consultations and interactions, and aided to prioritise issues of importance [10, 29, 37, 40, 42, 43, 50, 57] (see also "Attitudes to the care experience"). Some studies [31, 42, 48, 49] noted that HPs also saw PROMs as prompting patients to identify and prioritise issues from their own perspectives: "Actually, it [using the questionnaire] meant that we talked about issues which we wouldn't otherwise have touched upon because she hadn't thought of it, and I usually don't ask about it / ... / it 


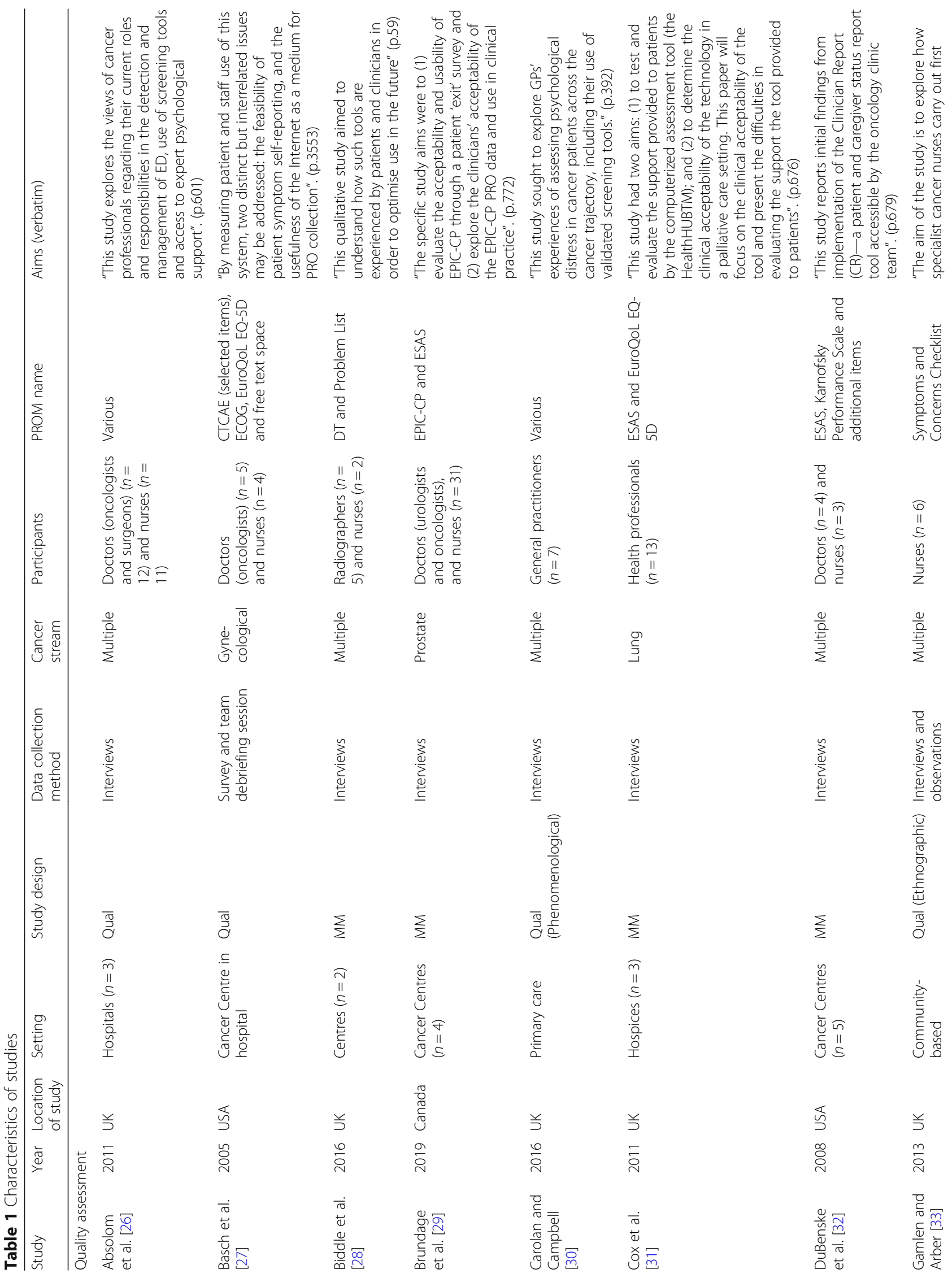




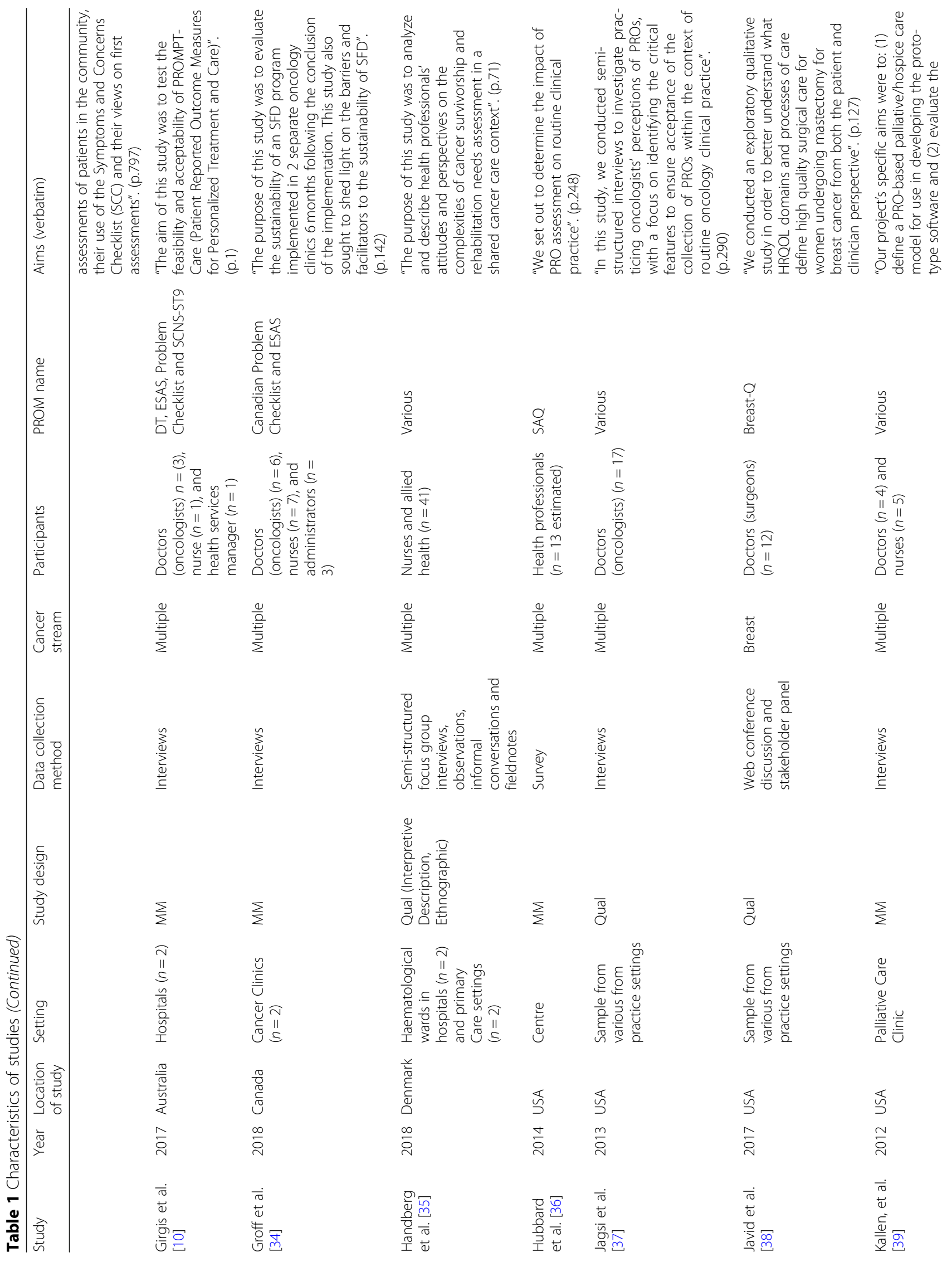




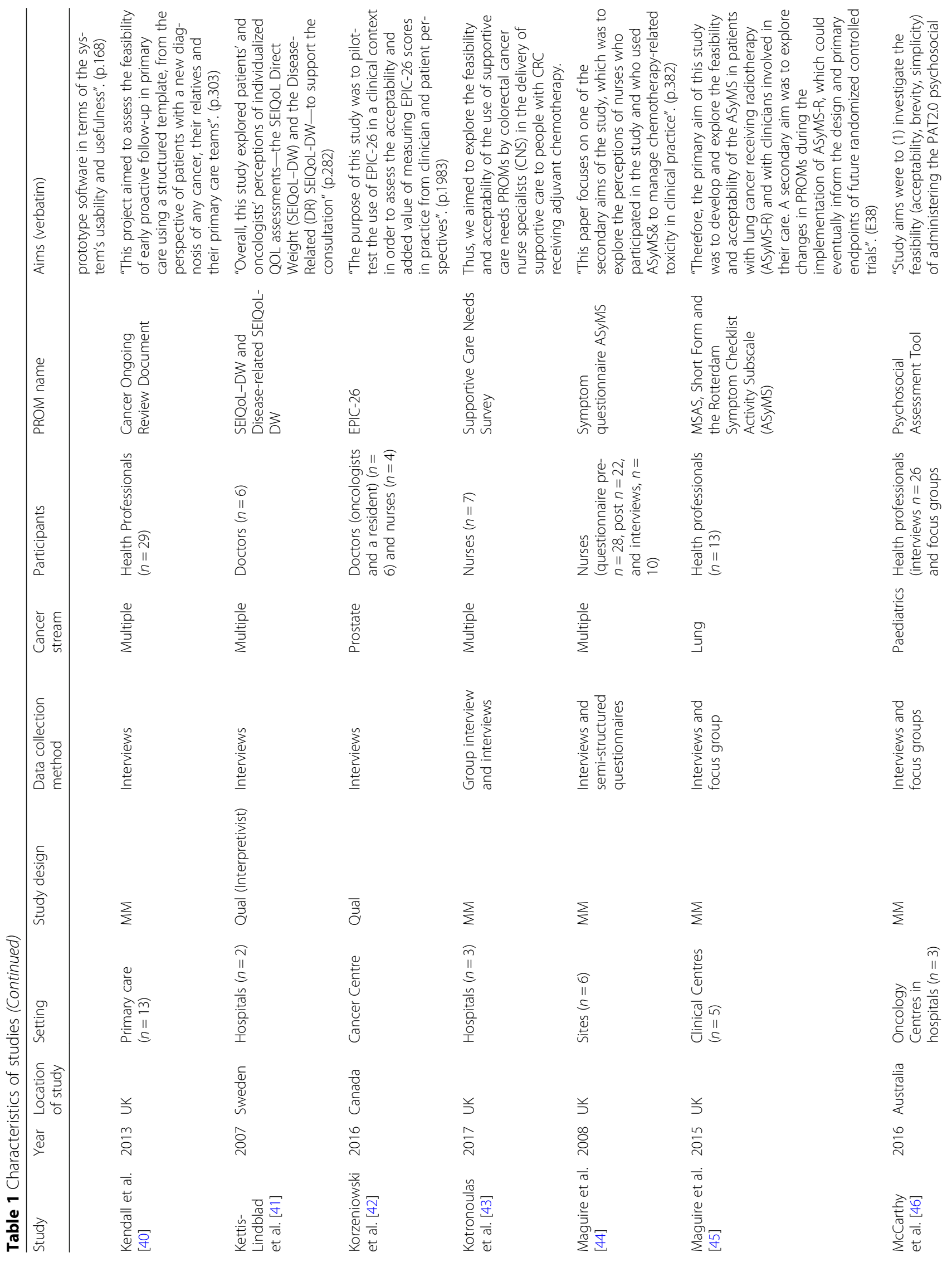




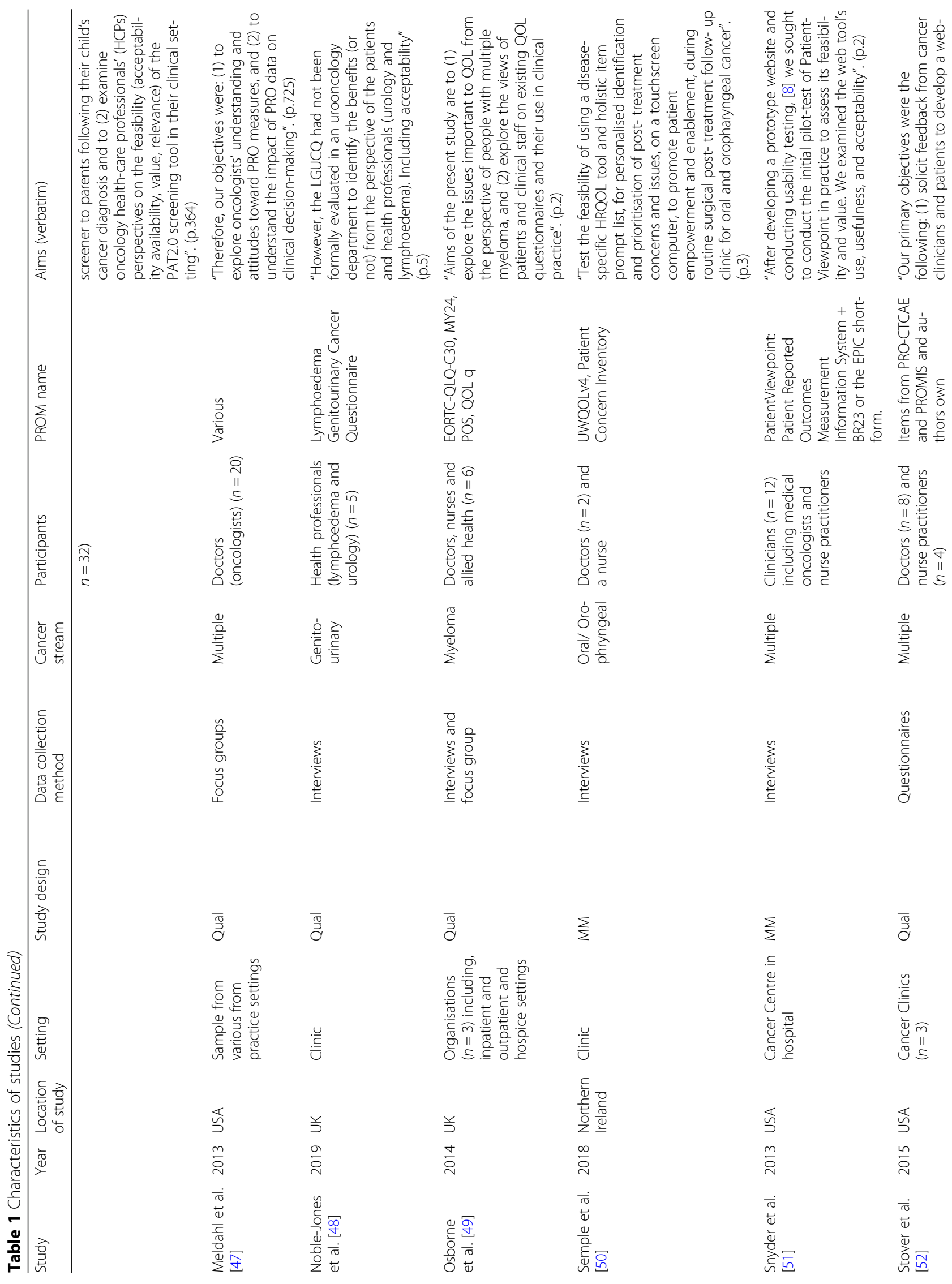




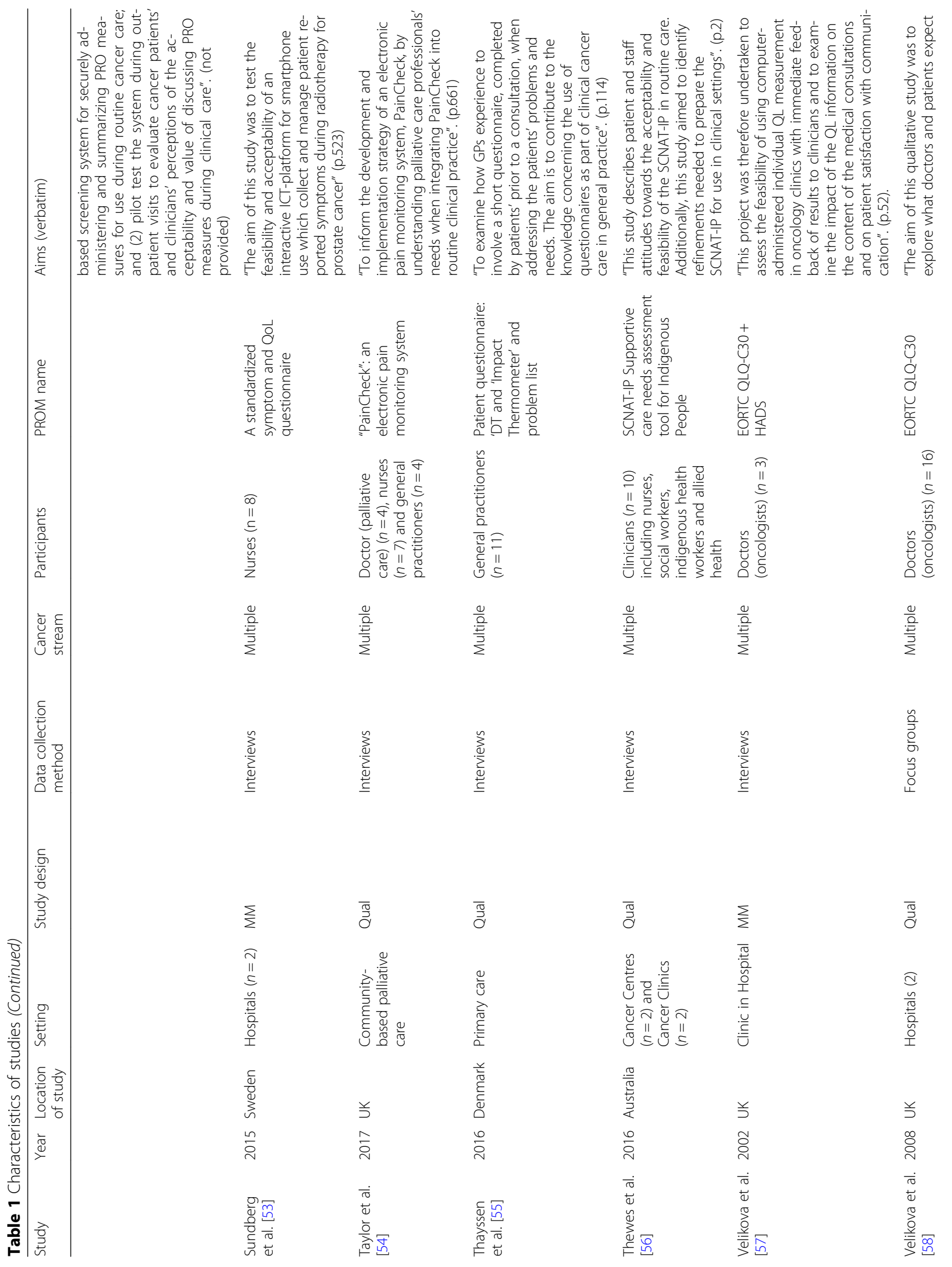




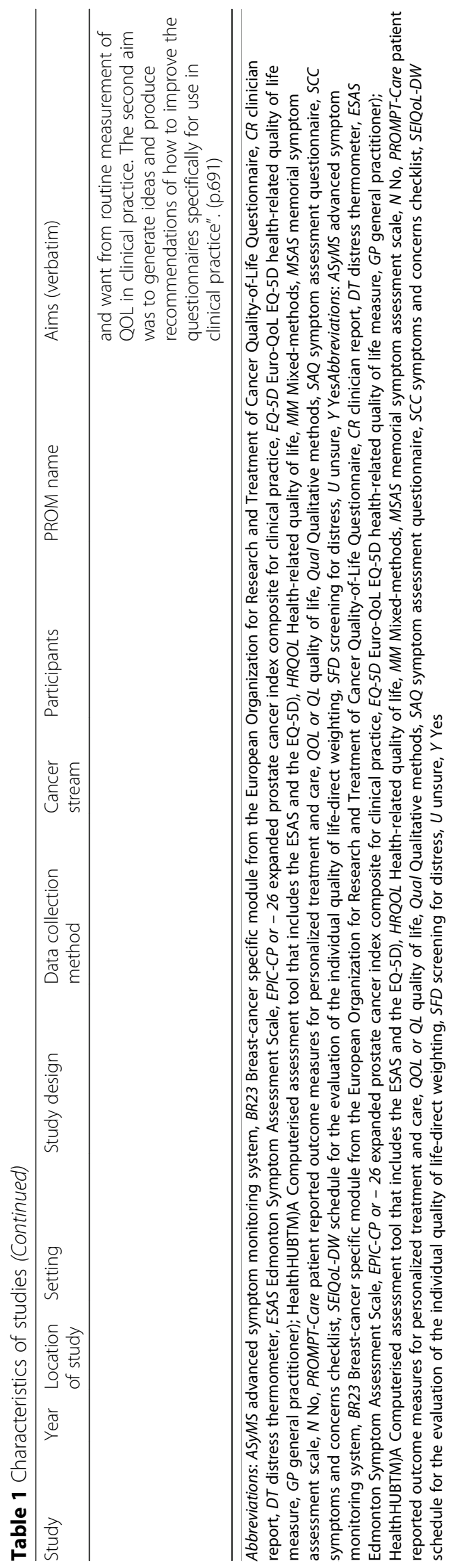




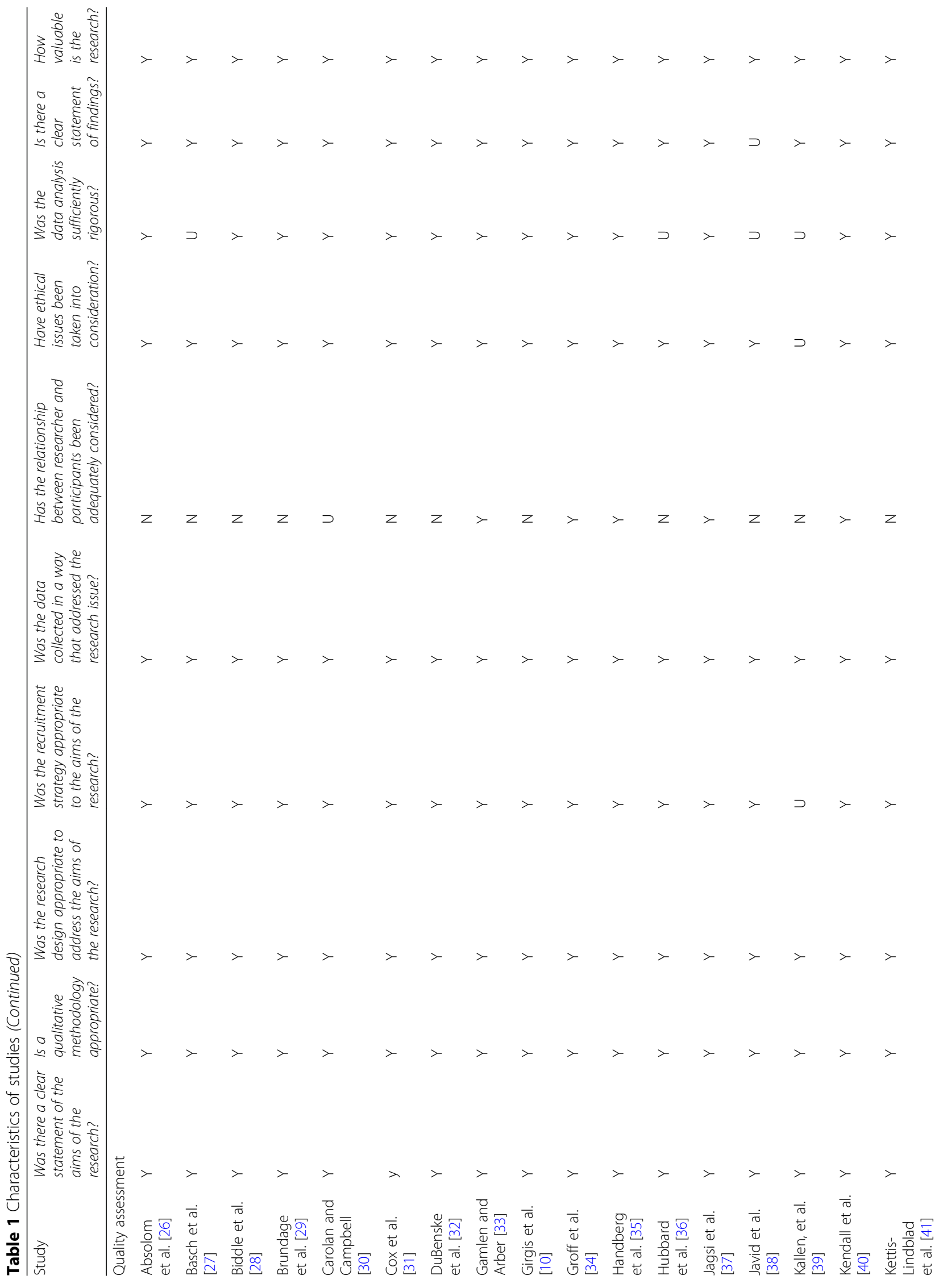




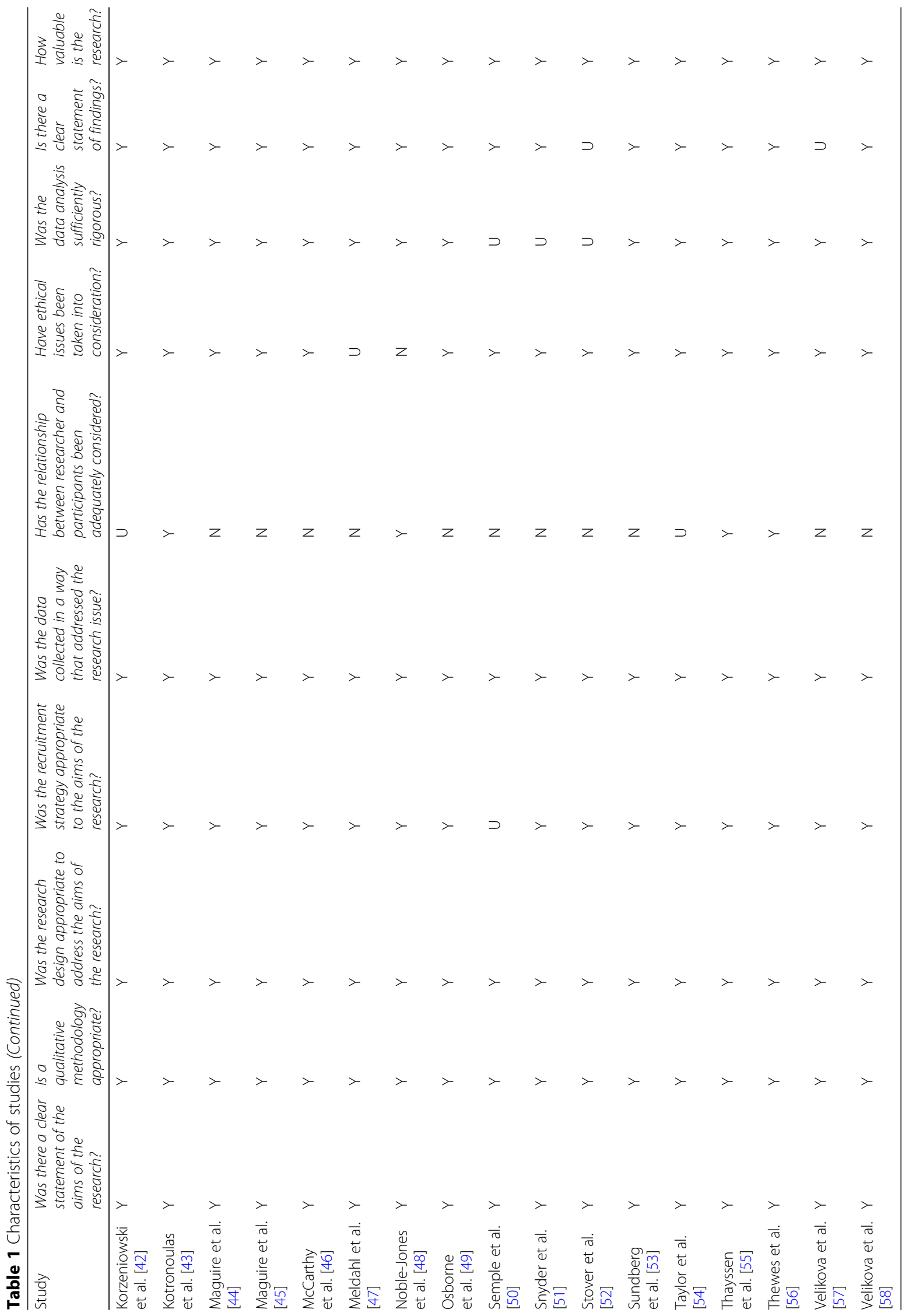




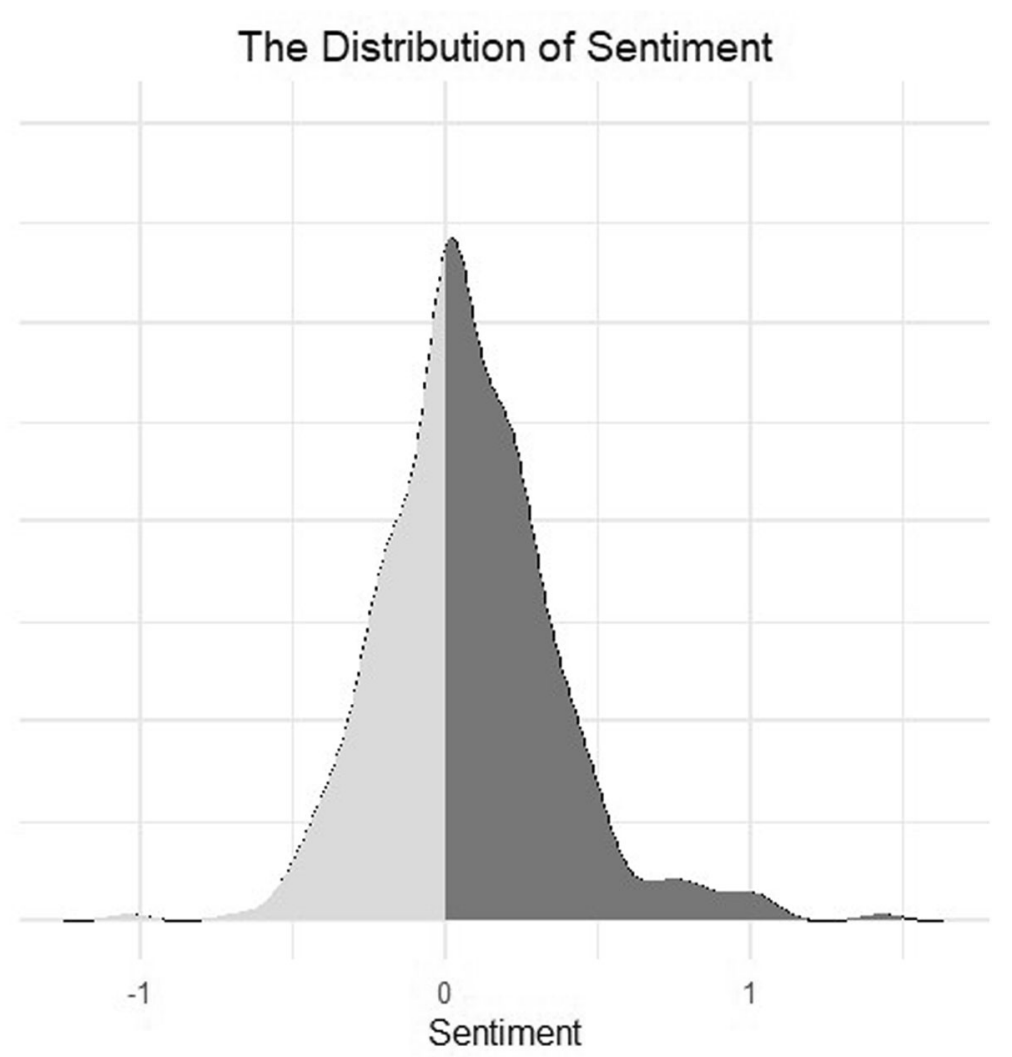

Fig. 2 Sentiment scores for HPs' attitudes towards PROMs

was actually quite important to her. It meant a lot to her" [55] (p117)

Sensitive issues A few studies $(n=6)$ reported that PROMs could "facilitate open dialogue and discussion of sensitive topics" [29] (p776), related to issues that could be potentially upsetting or distressing [33, 40, 43, 48]. It was noted that this may vary by patient: "I do think it's an important issue for patients, but obviously for some people it's an embarrassing one to bring up ... and it might be that you can't solve the problem ... but for some patients it's a very simple 'how many platelets do I need to have sex?" [49] (p11).

Distress or embarrassment may also be experienced by staff administering the PROMs [30, 33]. Only one study explored PROMs designed for Indigenous patients [56]; it highlighted the additional dimension of cultural sensitivity in the use of PROMs and professionals reported that the purpose-designed tool helped them to better connect with Indigenous patients [56].

Clinical usefulness of information Studies revealed widely differing views as to what was gained from PROMs data. Some findings suggested that PROMs brought new information to the fore $[33,35,41,46,48$,
$49,55,57$ ], while others believed this information was already collected through other means [10, 26, 32, 42, 51]. The perceived meaningfulness of PROMs data was linked to the relevance of the items collected to the disease and the needs at different points over the patient's journey [30, 37, 42-45, 52, 56, 57]; “patients' problems vary during treatment and follow-up" [58] (p694). For some, the thresholds for alerts and detection of change over time were clinically useful in the identification of issues [31, 32, 36, 44, 58].

Some studies $(n=8)$ reported that HPs regarded PROMs data as clinically valuable and felt that the patient was the best judge of their symptoms, regarding their responses to be accurate and honest [29, 31, 42, 46-48, 55, 57]. As reported: "I trust you [the patient], you tell me when you've got a problem, you tell me how you're getting on, here's this piece of technology to enable you to do that" [31] (p678).

In contrast, Basch et al. [27] reported that clinicians thought that patients overstated their symptoms, or believed patients had difficulty distinguishing between the levels of severity [56]. On this theme, other studies reported that HPs wanted what they considered to be more objective, valid and reliable information about symptoms [32, 38]. 


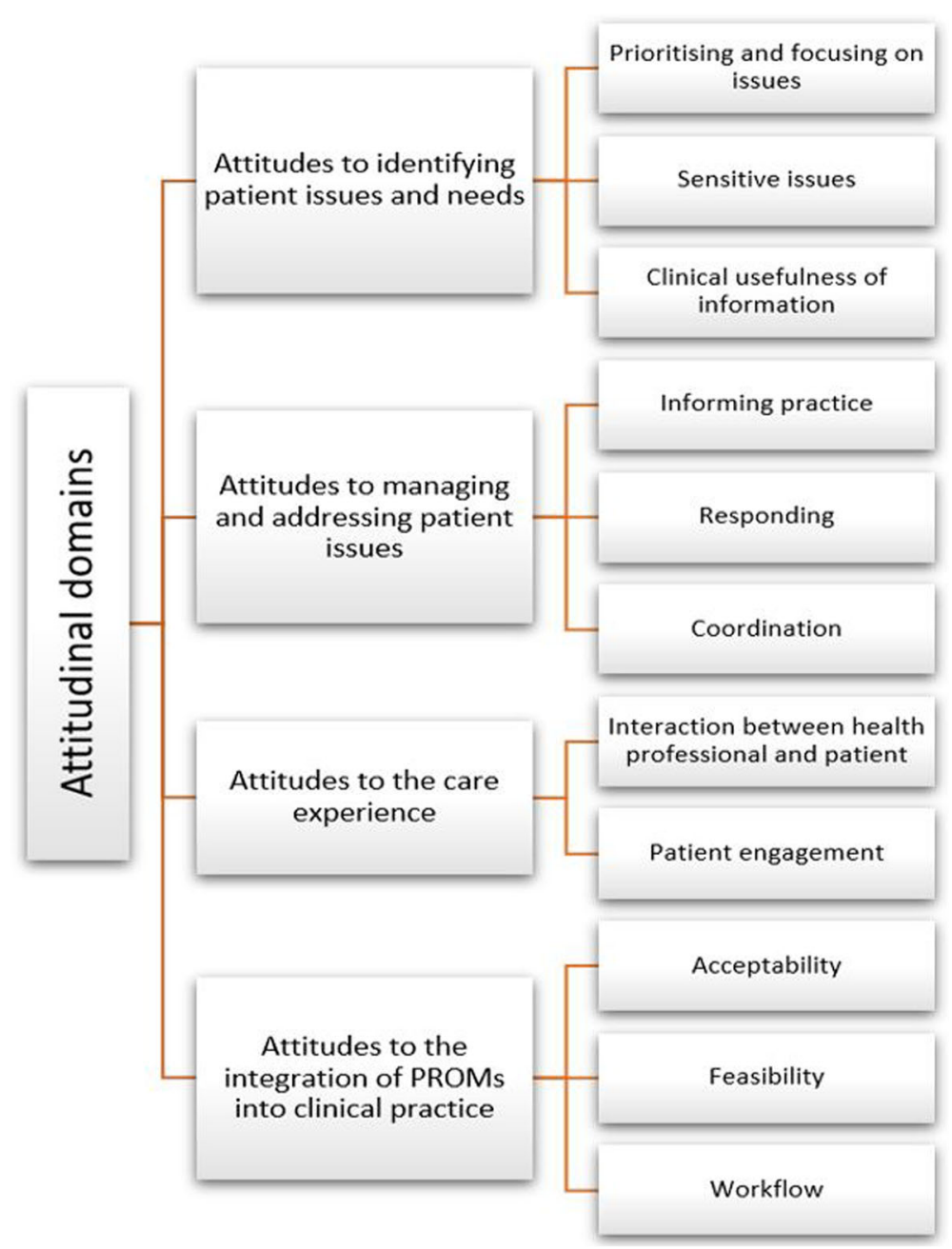

Fig. 3 Themes identified regarding HPs' attitudes towards PROMs

\section{Attitudes to managing and addressing patient issues}

This theme addresses what happens with needs that are identified.

Informing practice There were mixed views about whether PROMs would inform the practice of HPs. Some studies $(n=9)$ reported that PROMs helped HPs to ensure comprehensive coverage of issues and helped to refine the focus and streamline consultations $[29,41$, $42,48,49,51,53,54,58]$. HPs saw the potential for this to influence positively decision-making [27, 28, 31, 33, 42, 45, 49, 51, 52, 54]. In other cases, HPs felt PROMs information would have little impact on their practice or were uncertain about how much it influenced their patient management. Difficulties were also attributed to problems in interpretation of PROMs, to the timing of PROMs, the challenge of translating the data into the clinical domain and to a lack of guidance about how
PROMs data might be integrated into decision-making $[28,35,36,47,51,57,58]$.

Responding PROMs were viewed as generating a range of responses in patient management. Response strategies included: intervention where issues were identified [26, $27,32,35,46]$; adoption of a more holistic management approach [40, 42, 45, 50]; modification of communication approaches $[30,32,39,47,54,56]$; and the promotion of patient self-management $[44,53]$. Study findings also raised the fear that PROMs could bring up issues for which no adequate response existed, particularly in relation to financial difficulties, psychological issues and fatigue $[10,28,34,37]$ :

I find it very hard to discuss finances with patients, especially when it comes to, "they say I'm not entitled to any benefits", and I'm thinking, "well I can't do anything about that unfortunately". I feel 


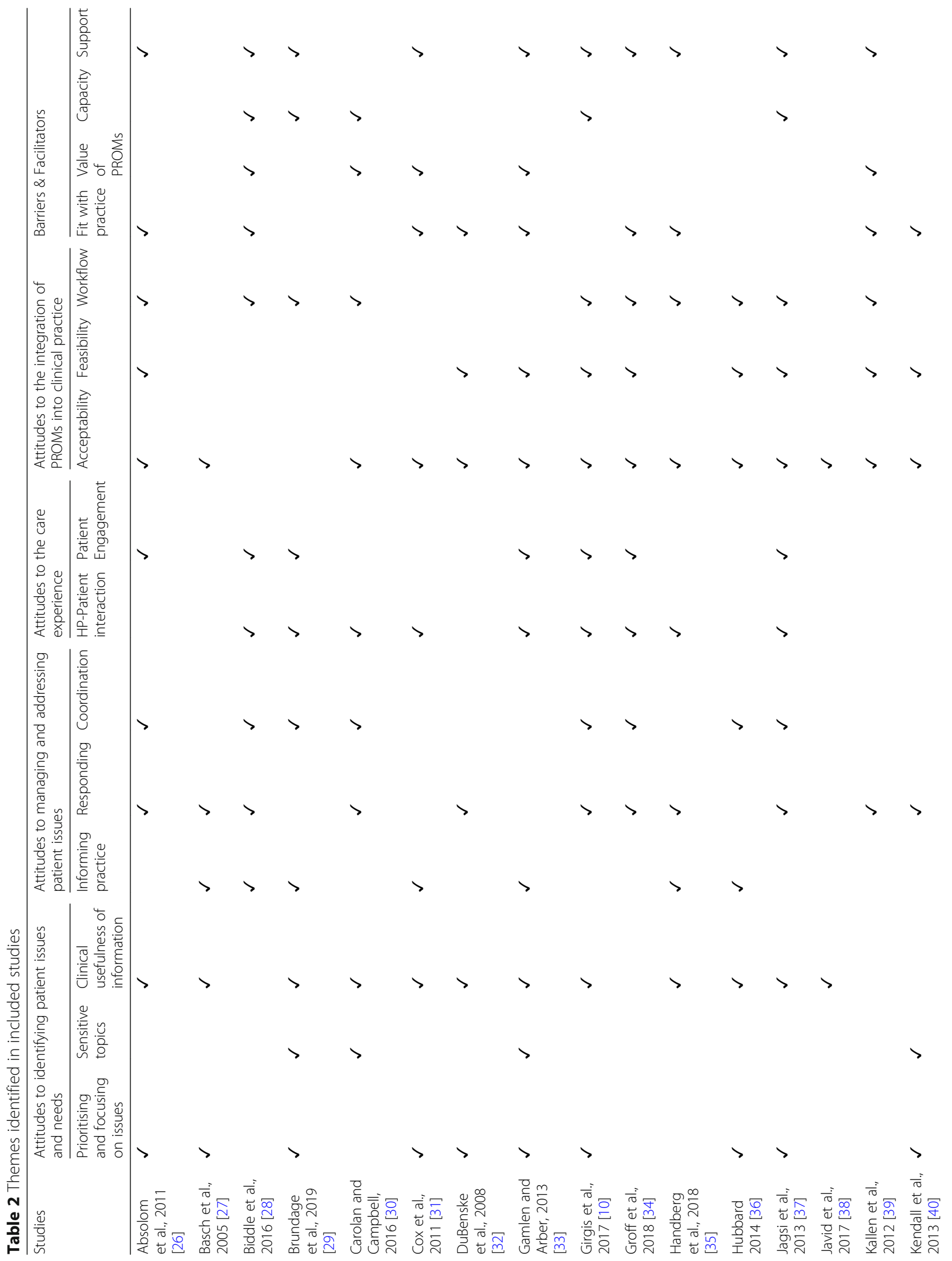




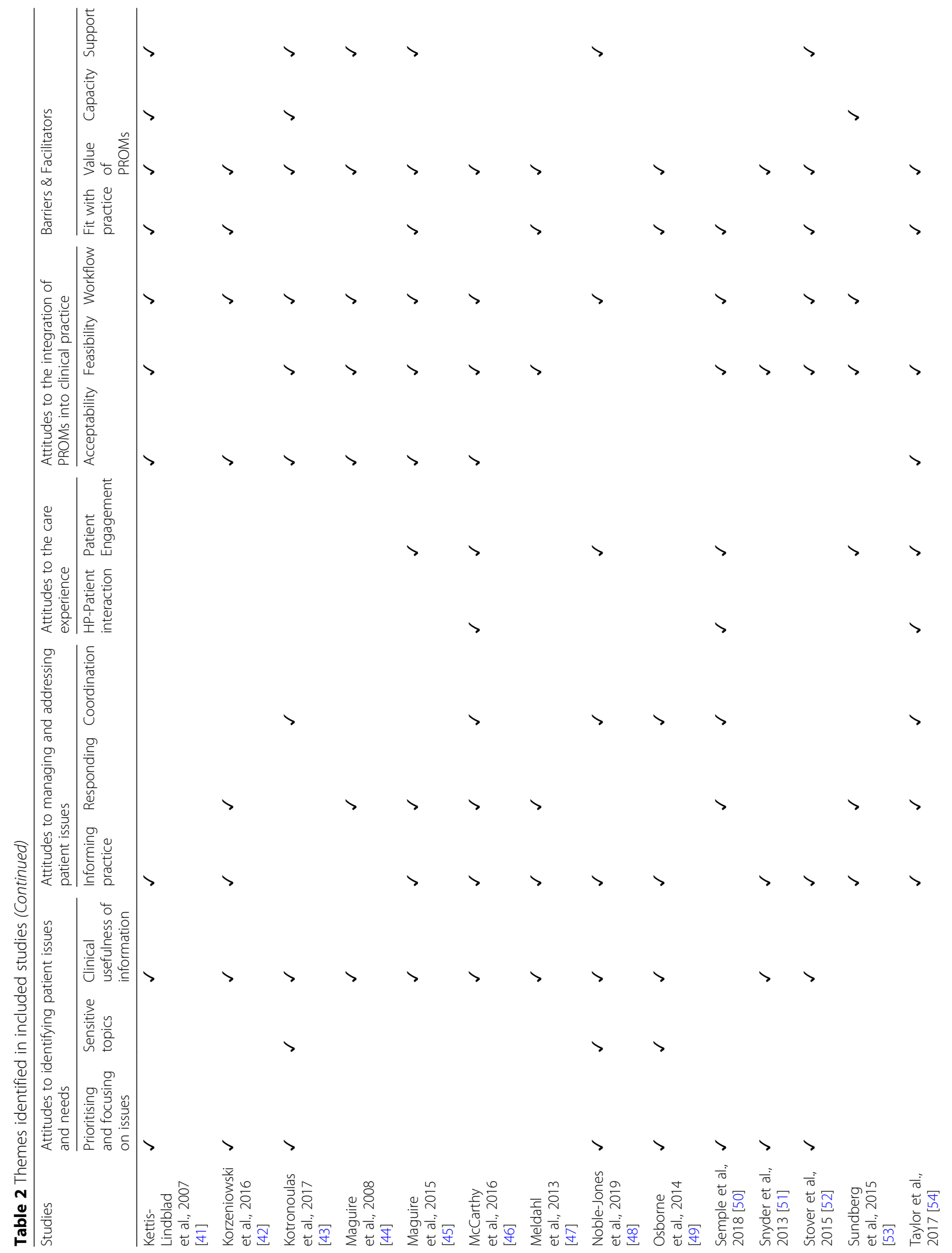




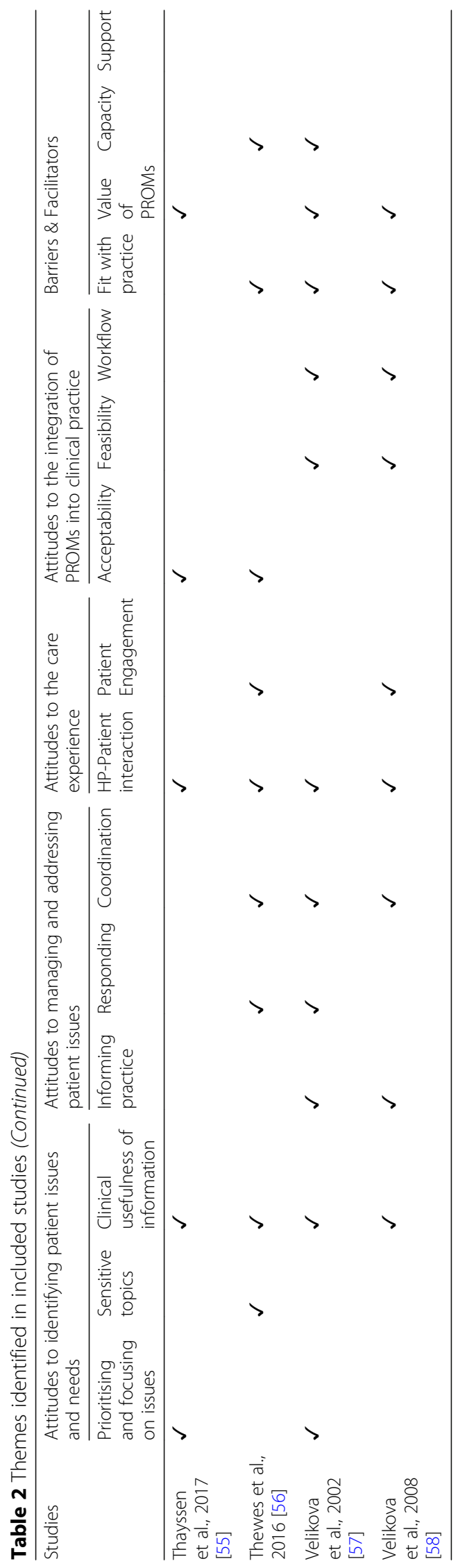


inadequate $\ldots$ because a patient could get a false sense from this thinking, "oh they can do something about it" ... if it's on there [PROMs] ... and you can't do anything about it [28] (p61).

Coordination Coordination underpinned the management of PROMs. Prompting referral to specialists outside of the care team (e.g. allied health) was cited as a key benefit of using PROMs [29, 30, 37, 43, 46, 48-50, $57,58]$. Some studies highlighted the importance of clarifying roles and responsibilities in order to coordinate the management of care, especially in relation to psychological and emotional issues [30, 34, 36, 37, 54, 56]. For example, in a number of studies, oncologists and surgeons felt that nursing staff were better positioned to engage with psychosocial issues, and in some studies nursing staff agreed [10, 26, 28].

\section{Attitudes to the care experience}

Several studies reported on oncology professionals' views of how PROMs may shape the experience of patient care.

Interaction between $\mathrm{HP}$ and patient An often-cited benefit of PROMs was that it facilitated communication between the HP and the patient [29, 46, 50, 56, 57], allowing staff to get to know patients better [10, 34, 46, 50, 55]. For example:

the participants reported that SFD [PROM] helped them connect with patients by facilitating conversations and encouraging patients to communicate their concerns. This was seen through comments such as "I think it's super helpful just to start conversations" and "It's opened the conversation for other things that may have been going on in their lives that we weren't seeing" [34] (p144).

In contrast, other studies raised the possibility that the use of PROMs might actually stifle rapportbuilding and diminish the "human touch" within the interaction $[28,30,31,33,35,37,54,58]$.

Patient engagement PROMs are perceived as a means through which patients may more actively direct their own care $[34,37,50]$. HPs in some studies $(n=7)$ described the "empowering" facets of PROMs-supported engagement, noting it could promote the "voice" of patients in their care $[29,34,37,45,48,50,56]$. A few studies $(n=3)$ also noted the potential for PROMs to bring patients back into care when they had unresolved issues $[10,53,54]$. Some HPs expressed concerns that completing PROMs adds to the burden placed on patients, especially where they perceived patients to not be well enough [28, 46, 58]. Several studies $(n=5)$ cited practical and logistical barriers limiting the completion of PROMs by patients or their carers [28, 33, 37, 56, 58]. For example, HPs noted that there were a number of patient factors, such as language proficiency, literacy level, technological aptitude (where relevant) and residential location which may make it challenging for certain patients to participate in PROMS $[26,28,37,56]$.

\section{Attitudes to the integration of PROMs into clinical practice}

The attitudes clinicians hold towards the integration of PROMs into practice is explored in this final theme.

Acceptability More than half of studies $(n=18)$ reported that HPs viewed PROMs to be acceptable, while noting that not all studies explicitly explored this. The acceptability was often linked with the perceived benefits described in the preceding themes, with an emphasis upon improving care and the experience for patients [10, 27, 31, 32, 34-37, 39-45, 55, 56]. Similarly, unacceptability derives from the challenges raised previously, such as, concerns about the relevance of a PROM, and issues relating to feasibility and the impact on workflow, as discussed below [26, 30, 33, 44, 46, 54].

Feasibility Studies highlighted the importance of how PROMs were integrated with existing systems and practice. Where PROMs were electronic, integration within the existing management system was viewed to enhance feasibility [34, 36, 40, 46, 50-52], but it was important that the PROMs were easy to navigate and that professionals were equipped with the computer access, knowledge and skills they would need [10, 37, 39, 41, 45, 47, 54]. The introduction of PROMs also represented a shift in practice which needed to be carefully integrated in relation to the setting culture and as was noted as a key concern of staff, "alongside clinical priorities" [26, 33, 43, $44,53,57,58]$ : "The participants indicated that there are 'at least a hundred other priorities that compete with SFD [PROM] and these priorities have the potential to threaten the sustainability of the program' and that SFD could fall off over time as other priorities emerge" [34] (p145).

Workflow The implications of PROMs on workflow was widely explored in the studies reviewed. A frequently expressed concern was that the collection and management of PROMs could create additional demands on staff [ $26,35,42,44-46,57,58]$. This was particularly worrying for HPs who believed that this information to be already collected via other means.

While on the one hand fears were raised that PROMs would require an increased work and time commitment from professionals to address identified needs [10, 34, 
$35,37,41,44,50,53]$, other HPs noted that PROMs presented opportunities for efficiency and time-svaing [29, 36, 39, 48, 50]. For example, where PROMs were regarded as focusing interactions (see "prioritising and focusing on issues"), this could enhance efficiency, as it would allocate attention to prioritised issues [29]. The introduction of PROMs into the existing flow of work was viewed as potentially challenging, but it was recognised that increasing familiarity would facilitate integration $[43,52]$.

Clarity concerning the roles and responsibilities of team members (e.g., who should manage psychosocial issues) [28], emerged as an important issue for managing workflow $[28,30]$. There is also a recognition that certain key personnel may be over-extended as a result of PROMs: "Oncologists perceived the CNS [Clinical Nurse Specialist] as a 'crutch' for patients but also appreciate they are a stretched resource" [26] (p604).

\section{Barriers and facilitators to the uptake of PROMS}

A number of factors were found to impede or promote the adoption and use of PROMs. These centred around: their fit with existing practice; how PROMs were valued; the capacity of professionals to respond to PROMs; and the support in place.

\section{Fit with practice}

Perceptions that PROMs would not align with or would disrupt existing practice were identified as a barrier to uptake, especially where this was viewed as an additional task on top of other competing demands [26, 32, 34, 35, 40, 41, 56, 57]. Embedding PROMs within the existing systems, frameworks and practice could act as a facilitator: "For the future, the introduction and implementation of an assessment tool should be embedded within the total assessment process" [33] (p801).

Guidelines for the use of PROMs in practice could facilitate integration $[28,52,57]$. For electronic PROMs, a lack of fit within the existing patient management system formed a barrier (e.g. if it operated separately to this) $[31,32,34,40,45,52]$, while full embedding within the patient management system and ease of use were enabling factors [32, 34, 39, 40, 42, 47-50, 58].

\section{Value of PROMs}

HPs' perceptions about the value of PROMs may shape their uptake and use. A negative perception of the clinical usefulness of PROMs may be a barrier, while positive perceptions may promote use. The perceived relevance, specificity and evidence for the PROMs introduced is therefore key [28, 31, 33, 41$47,49,51,52,57,58]$. Another dimension of value related to whether HPs viewed PROMs to positively impact patients, with a lack of perceived benefit forming a barrier [30, 39, 54, 55, 58].

\section{Capacity}

Barriers emerge where professionals did not feel there was capacity to respond to PROMs for a range of reasons including self-efficacy, access to specialised knowledge, management arrangements within the team or the nature of the issues raised [10, 28-30, 37, 41, 43, 53, 56, 57]: Once you've had the interview and you say, "right, okay, I'll go and make these phone calls", ... "right, ok, I've got to do this, I've got to do that", and no added time was given. [It's] the aftermath as well ... if they'd allowed us more time, I think it could have been more effective [28] (p63).

\section{Support}

Training, practice opportunities and ongoing support would help to facilitate the adoption of PROMs [10, 26, 28, 29, 31, 33, 34, 39, 41, 43-45, 48, 52]. Additionally, appreciation on the part of managers about the impacts of PROMs and the involvement and "buy in" from frontline staff could support implementation [34, 35, 37].

\section{Discussion}

We systematically reviewed qualitative evidence to enhance understanding of HPs' attitudes towards PROMs in oncology services, and to gain insight into the barriers and facilitators for their adoption and use. Our metasynthesis identified four key attitudinal domains: identifying patient issues and needs using PROMs; managing and addressing patient issues; the care experience; and the integration of PROMs into clinical practice. Key considerations for implementation of PROMS include: the fit with existing practice; how PROMs are valued; the capacity of professionals to respond to PROMs; and the supports in place.

PROMs have received increased attention from oncology researchers $[4,5]$. Overall, the qualitative evidence is of good quality and captured recent findings, with half of the studies having been published from 2015 onwards. The inclusion of qualitative content from mixed-method research, where appropriate, facilitated examination of findings from study designs that appear to be often employed in research on the development and piloting of PROMs. The majority of studies are based in North American and European contexts, and encompass a range of service settings where PROMs may be applied, as well as presenting the views of various professional groups involved in their use. In many cases, studies included well-established patient measures, but also included views about PROMs use as a general concept.

The overall sentiments from the opinions of HPs towards PROMs was mostly neutral, with a more positive 
tail compared to negative densities. Consistent with the literature, many HPs believe PROMs support communication with patients; enabling them to know their patients better $[4,5,43]$ but there are concerns that PROMs could negatively impact these relationships by diminishing the "human touch" in the care process [15]. In relation to sensitive or embarrassing issues for patients, PROMs were viewed as both potentially facilitative and potentially upsetting; this reflects previous findings $[3,8]$. Interestingly, a few studies reported that embarrassment or distress may also be experienced by HPs using PROMs [30, 33]. One study included indepth considerations of the cultural dimensions of PROMs, in which largely favourable views were expressed by HPs about the use of culturally appropriate PROMs designed for care of patients from Australian Indigenous communities [56]. We would advocate for further exploration of PROMs development and use in the care of patients from culturally and linguistically diverse communities, especially as our analysis noted that HPs viewed patients' language proficiency as a potential impediment to meaningful participation.

Many HPs perceived PROMs as facilitating the identification of patient issues, including non-medical concerns such as psychosocial and quality of life issues [3, 4, 12, 15]. Some HPs see PROMs as empowering patients by encouraging them to give voice to issues they experience as significant and facilitating their capacity to play a more active role in their care, including through selfmanagement (c.f. Howell et al. [3]). However, staff also gave careful thought to logistical and practical barriers that patients may face in reporting PROMs, and to the burden this could place on patients. The capacity for these measures to positively affect patients' care was a key element of HPs' appraisal of the value of PROMs; it is a likely a critical determinant of the active uptake of PROMs in practice.

The nature and timing of the PROMs data to be collected are important. In alignment with previous reports, HPs placed value on the collection of data which was: novel (i.e. avoiding duplication); relevant, containing clear indicators of clinical significance; appropriate for the specific cancer; and collected at significant points in the patient journey. These valuations were interwoven with HPs' views about how these PROMs data would be subsequently used in practice. Perceptions of poor clinical utility could form an implementation barrier. HPs recognised the potential to promote earlier intervention and more holistic approaches to care but were concerned where they felt unable to adequately respond to the issues identified (e.g., issues for which feasible solutions were unavailable).

Some studies reported that HPs were concerned that some patients may have difficulty distinguishing levels of severity and therefore they desired more objective measures $[27,32,38,56]$. A clinician with these concerns, overall or for a specific patient, may find it challenging to know how to best proceed. This mix of positive views and negative fears is perhaps not surprising, as previous reviews have not been able to establish conclusively the impact of PROMs on corresponding clinical actions, such as an increase in appropriate referrals or changes to patient management plans $[5,8,13]$.

Howell et al. [3] observed that the relationship between the clinical communication of PROMs data and health outcomes is complex. This meta-synthesis of professional end-users' accounts can contribute to deepening understanding surrounding the potential for disjointedness. Staff members' views about their capacity to respond to PROMs data are crucial: they need the skills to engage with patients about needs identified by PROMs, including the skills and confidence to discuss sensitive subjects; they need to be comfortable that they have sufficient time to respond, given competing priorities in their workload; and they need to have referral pathways for patients whose needs require additional time or expertise. As coordination may bring challenges, the clear delineation of roles and responsibilities associated with responding to PROMs data within care teams may help to optimise a response. Further, recognition of any additional time that may be needed for the management of PROMs data, including accounting for additional time during the initial learning curve could facilitate active uptake. This may be especially important where HPs need to develop additional technological skills.

Findings from Chen et al.'s [5] systematic review suggest that the reporting of data alone may not be sufficient, but that positive changes in patient management could be more probable where PROMs collection is integrated into care planning. Our findings echo this principle and point to several priority areas for integration. From the accounts of HPs, PROMs use could be facilitated where it is embedded within the existing patient management system, and where staff are able to navigate this with ease. In this regard, the electronic collection of PROMs may have a distinct advantage. It was also deemed important that PROMs be incorporated within existing practices (e.g. consultations or assessment practices) and this could optimise efficiency. Providing staff members with guidelines for the use of PROMs in clinical practice may also be beneficial. Consistent with the literature $[4,5,13,15]$, our analysis would suggest that implementation efforts could be enhanced by the provision of staff training and ongoing support, establishing "buy in" from front line staff and appreciation on the part of managers about how PROMs may impact clinical practice. However, many of the issues raised such as coordination, workflow and clinical utility, 
which form negative HP attitudes are vital to tackle alongside these recommendations to improve implementation prospects.

\section{Limitations}

The search was restricted to four databases, included only English language records and excluded grey literature. It is therefore possible that relevant studies may have been overlooked. We deemed it informative to include qualitative findings from mixed-method designs as well as qualitative studies. This resulted in the inclusion of studies with a range of methods and varying proportions of qualitative research (e.g. the qualitative content might have only represented a minority component of a mixed-method study). We sought to manage this through applying a criterion for studies to have analysed and reported on qualitative content separately, in addition to employing a uniform quality assessment procedure. The qualitative evidence comes from studies conducted in diverse health service contexts, including multi-centre studies. As such, the general concerns about de-contextualisation and over-extrapolation in qualitative meta-synthesis required careful consideration in the process of synthesis and analysis $[59,60]$. We purposefully selected a strategy that involved an iterative cycle of engagement with the extracted data as part of the analysis process and was grounded in an approach successfully used with a large data set of qualitative studies [24].

Our study excludes the attitudes of patients and thus may miss some important PROMs implementation information. However, following initial scoping of the relevant literature, we deemed it necessary to narrow the scope so to ensure sufficiently comprehensive engagement with the substantial and diverse HP evidence base. Similarly, we would consider patient attitudes towards PROMs in oncology to warrant dedicated and in-depth study. Further, it is a limitation for the sentiment analysis that results could only be obtained from primary text available in the manuscripts that were synthesised and not all the qualitative data that were collected in these studies. Most qualitative manuscripts present only representative quotes, and often these consist of positive and negative representatives. Our neutral finding in the sentiment analysis may be due to this limitation, where a balanced representation was provided from the papers.

\section{Conclusion}

The use of PROMs in oncology services may offer a valuable tool to help identify and manage patients' needs over the course of their cancer journey. This paper advances existing understanding by reporting on a body of evidence which captures the sentiments and perspectives of those end-users critical to the adoption of PROMs in oncology practice. The findings articulate important pre- conditions, from the perspectives of HPs, for the successful implementation of PROMs; providing insights for policy-makers and services involved in rolling out these initiatives. Examination of the evidence points to several future research directions. Further exploration of the potentially empowering dimension of PROMs use could begin with a meta-synthesis of the qualitative evidence concerning oncology patients' experiences with PROMs. The accessibility of PROMs for patients, including those from culturally and linguistically diverse communities would be valuable to synthesise. Implementation efforts could be enhanced through greater knowledge of the resources and care processes needed to support the translation of collected PROMs data into corresponding actions, to the benefit of patients.

\section{Supplementary information}

Supplementary information accompanies this paper at https://doi.org/10. 1186/s12913-020-4939-7.

Additional file 1. PRISMA Checklist.

Additional file 2. List of PROMs abbreviations.

Acknowledgements

Not applicable.

Authors' contributions

The review was conceived of by $Y T, B N G E, M B$ and $G A$ and the study design was developed by $Y T, B N G E, M B$ and GA in consultation with JB. BNGE, YT, $M B$ and $G A$ undertook systematic searching and review. BNGE and MB undertook the qualitative analysis and $Y T$ conducted the sentiment analysis. GPD, WL, RLW, IO, DC, AG, ID and JB made important contributions to the interpretation of the data. BNGE produced the first draft of the paper and $Y T$, $M B, G A, G P D, W L, R L W, I O, D C, A G, I D$ and JB provided critical and substantial revisions to the manuscript. BNGE, YT, MB, GA, GPD, WL, RLW, IO, $D C, A G, I D$ and JB approved the final version of the manuscript.

\section{Funding}

JB receives funding to support this work from multiple Australian National Health and Medical Research Council grants, e.g., APP9100002 and APP1135048. The funding body had no direct role in the design of the study, in the collection, analysis, and interpretation of data or in writing the manuscript.

Availability of data and materials

Articles included in the analysis are cited in the reference list.

Ethics approval and consent to participate

Not applicable.

Consent for publication

Not applicable.

Competing interests

The authors declare that they have no competing interests.

\section{Author details}

${ }^{1}$ Australian Institute of Health Innovation, Macquarie University, North Ryde, NSW 2109, Australia. 'Liverpool Cancer Therapy Centre, Liverpool, NSW 2170, Australia. ${ }^{3}$ South Western Sydney Clinical School, University of New South Wales, Liverpool, NSW 2170, Australia. ${ }^{4}$ Centre for Oncology Education and Research Translation (CONCERT), Ingham Institute for Applied Medical Research, Liverpool, NSW 2170, Australia. ${ }^{5}$ St. George Cancer Care Centre, St. George Hospital, Kogarah, NSW 2217, Australia. ${ }^{6}$ St. George Hospital Clinical 
School, University of New South Wales, Sydney, NSW 2217, Australia. ${ }^{7}$ Faculty of Medicine and Health, The University of Sydney, Sydney, NSW 2006, Australia. ${ }^{8}$ Prince of Wales Clinical School, University of New South Wales, Sydney, NSW 2052, Australia. ${ }^{9}$ Faculty of Health and Medical Sciences, University of Adelaide, Adelaide, SA 5000, Australia. ${ }^{10}$ College of Medicine and Public Health, Flinders University, Adelaide, SA 5042, Australia. ${ }^{11}$ Faculty of Health, University of Technology Sydney, Sydney, NSW 2007, Australia.

Received: 18 November 2019 Accepted: 28 January 2020 Published online: 10 February 2020

\section{References}

1. Basch E, Deal AM, Kris MG, Scher HI, Hudis CA, Sabbatini P, et al. Symptom monitoring with patient-reported outcomes during routine cancer treatment: a randomized controlled trial. J Clin Oncol. 2016;34(6):557-65.

2. Luckett T, Butow PN, King MT. Improving patient outcomes through the routine use of patient-reported data in cancer clinics: future directions. Psychooncology. 2009;18(11):1129-38.

3. Howell D, Wilkinson K, Molloy S, Green E, Liberty J, Orchard K, et al. Patientreported outcomes in routine cancer clinical practice: a scoping review of use, impact on health outcomes, and implementation factors. Ann Oncol. 2015;26(9):1846-58.

4. Williams K, Sansoni J, Morris D, Grootemaat P, Thompson CJ. Patientreported outcome measures: literature review. Sydney: Australian Health Services Research Institute, University of Wollongong; 2016.

5. Chen J, Ou L, Hollis SJ. A systematic review of the impact of routine collection of patient reported outcome measures on patients, providers and health organisations in an oncologic setting. BMC Health Serv Res. 2013; 13(1):211.

6. Newell S, Sanson-Fisher RW, Girgis A, Bonaventura A. How well do medical oncologists' perceptions reflect their patients' reported physical and psychosocial problems? Cancer. 1998;83(8):1640-51.

7. Nyrop KA, Deal AM, Reeder-Hayes KE, Shachar SS, Reeve BB, Basch E, et al. Patient-reported and clinician-reported chemotherapy-induced peripheral neuropathy in patients with early breast cancer: current clinical practice. Cancer. 2019;125(17):2945-54.

8. Kotronoulas G, Kearney N, Maguire R, Harrow A, Domenico DD, Croy S, et al. What is the value of the routine use of patient-reported outcome measures toward improvement of patient outcomes, processes of care, and health service outcomes in cancer care? A systematic review of controlled trials. J Clin Oncol. 2014;32(14):1480-501.

9. Girgis A, Durcinoska I, Koh E-S, Ng W, Arnold A, Delaney GP, et al. Development of health pathways to standardize Cancer care pathways informed by patient-reported outcomes and clinical practice guidelines. JCO Clin Cancer Inform. 2018;2:1-13.

10. Girgis A, Durcinoska I, Levesque JV, Gerges M, Sandell T, Arnold A, et al. eHealth system for collecting and utilizing patient reported outcome measures for personalized treatment and care (PROMPT-care) among cancer patients: mixed methods approach to evaluate feasibility and acceptability. J Med Internet Res. 2017;19(10):e330.

11. Ahmed S, Berzon RA, Revicki DA, Lenderking WR, Moinpour CM, Basch E, et al. The use of patient-reported outcomes (PRO) within comparative effectiveness research: implications for clinical practice and health care policy. Med Care. 2012;50(12):1060-70.

12. Valderas JM, Kotzeva A, Espallargues M, Guyatt G, Ferrans CE, Halyard $M Y$, et al. The impact of measuring patient-reported outcomes in clinical practice: a systematic review of the literature. Qual Life Res. 2008;17(2):179-93.

13. Greenhalgh J, Meadows K. The effectiveness of the use of patient-based measures of health in routine practice in improving the process and outcomes of patient care: a literature review. J Eval Clin Pract. 1999;5(4): 401-16.

14. Bottorff $\mathrm{J}$. Knowledge translation: where are the qualitative health researchers? Qual Health Res. 2015;25(11):1461-2.

15. Boyce MB, Browne JP, Greenhalgh J. The experiences of professionals with using information from patient-reported outcome measures to improve the quality of healthcare: a systematic review of qualitative research. BMJ Qual Saf. 2014;23(6):508-18.

16. Clarivate Analytics. Endnote X9. Jersey, British Isles. 2018. Available from: https://endnote.com/. Accessed Oct 2018.
17. Moher D, Liberati A, Tetzlaff J, Altman DG, Group P. Preferred reporting items for systematic reviews and meta-analyses: the PRISMA statement. PLoS Med. 2009;6(1):e1000097.

18. Critical Appraisal Skills Programme. CASP qualitative checklist. 2018. Available from: https://casp-uk.net/casp-tools-checklists. Accessed Sept 2018.

19. Pang B, Lee L. Opinion mining and sentiment analysis. Found Trends In Ret. 2008;2(1-2):1-135

20. R Core, Team. R: A language and environment for statistical computing. Vienna: R Foundation for Statistical Computing; 2013. Available from: http:// www.R-project.org/. Accessed June 2018.

21. Team R. RStudio: Integrated Development for R. Boston RStudio Inc. 2015. Available from: http://www.rstudio.com/

22. Rinker T. Package 'sentimentr'. 2019. Available from: http://github.com/ trinker/sentimentr

23. Mudge S, Kayes N, McPherson K. Who is in control? Clinicians' view on their role in self-management approaches: a qualitative metasynthesis. BMJ Open. 2015;5(5):e007413.

24. Thomas J, Harden A. Methods for the thematic synthesis of qualitative research in systematic reviews. BMC Med Res Methodol. 2008;8(1):45.

25. QSR International Pty Ltd. NVivo qualitative data analysis software. 2012. Available from: https://www.qsrinternational.com/nvivo/home

26. Absolom K, Holch P, Pini S, Hill K, Liu A, Sharpe M, et al. The detection and management of emotional distress in cancer patients: the views of healthcare professionals. Psychooncology. 2011;20(6):601-8.

27. Basch E, Artz D, Dulko D, Scher K, Sabbatini P, Hensley M, et al. Patient online self-reporting of toxicity symptoms during chemotherapy. J Clin Oncol. 2005;23(15):3552-61.

28. Biddle L, Paramasivan S, Harris S, Campbell R, Brennan J, Hollingworth W. Patients' and clinicians' experiences of holistic needs assessment using a cancer distress thermometer and problem list: a qualitative study. Euro J Oncol Nurs. 2016;23:59-65.

29. Brundage MD, Barbera L, McCallum F, Howell DM. A pilot evaluation of the expanded prostate cancer index composite for clinical practice (EPIC-CP) tool in Ontario. Qual Life Res. 2019;28(3):771-82.

30. Carolan CM, Campbell K. General practitioners' 'lived experience' of assessing psychological distress in cancer patients: an exploratory qualitative study. Eur J Cancer. 2016;25(3):391-401.

31. Cox A, Illsley M, Knibb W, Lucas C, O'Driscoll M, Potter C, et al. The acceptability of e-technology to monitor and assess patient symptoms following palliative radiotherapy for lung cancer. Palliat Med. 2011;25(7): 675-81.

32. DuBenske LL, Chih M-Y, Dinauer S, Gustafson DH, Cleary JF. Development and implementation of a clinician reporting system for advanced stage cancer: initial lessons learned. J Am Med Inform Assoc. 2008;15(5):679-86.

33. Gamlen $E$, Arber A. First assessments by specialist cancer nurses in the community: an ethnography. Euro J Oncol Nurs. 2013;17(6):797-801.

34. Groff S, Holroyd-Leduc J, White D, Bultz BD. Examining the sustainability of screening for distress, the sixth vital sign, in two outpatient oncology clinics: a mixed-methods study. Psychooncology. 2018;27(1):141-7.

35. Handberg C, Thorne S, Maribo T. When a policy decision meets practice realities: the case of cancer survivorship care and rehabilitation needs assessment. Euro J Oncol Nurs. 2018;33:70-7.

36. Hubbard JM, Grothey AF, McWilliams RR, Buckner JC, Sloan JA. Physician perspective on incorporation of oncology patient quality-of-life, fatigue, and pain assessment into clinical practice. J Oncol Pract. 2014;10(4):248-53.

37. Jagsi R, Chiang A, Polite BN, Medeiros BC, McNiff K, Abernethy AP, et al. Qualitative analysis of practicing oncologists' attitudes and experiences regarding collection of patient-reported outcomes. J Oncol Pract. 2013;9(6):e290-e7.

38. Javid SH, Lawrence SO, Lavallee DC. Prioritizing patient-reported outcomes in breast cancer surgery quality improvement. Breast J. 2017;23(2):127-37.

39. Kallen MA, Yang D, Haas N. A technical solution to improving palliative and hospice care. Support Care Cancer. 2012;20(1):167-74.

40. Kendall M, Mason B, Momen N, Barclay S, Munday D, Lovick R, et al. Proactive cancer care in primary care: a mixed-methods study. Fam Pract. 2013;30(3):302-12.

41. Kettis-Lindblad Å, Ring L, Widmark E, Bendtsen P, Glimelius B. Patients' and doctors' views of using the schedule for individual quality of life in clinical practice. J Support Oncol. 2007;5(6):281-97.

42. Korzeniowski M, Kalyvas M, Mahmud A, Shenfield C, Tong C, Zaza K, et al. Piloting prostate cancer patient-reported outcomesin clinical practice. Support Care Cancer. 2016;24(5):1983-90. 
43. Kotronoulas G, Papadopoulou C, MacNicol L, Simpson M, Maguire R Feasibility and acceptability of the use of patient-reported outcome measures (PROMs) in the delivery of nurse-led supportive care to people with colorectal cancer. Euro J Oncol Nurs. 2017;29:115-24.

44. Maguire R, McCann L, Miller M, Kearney N. Nurse's perceptions and experiences of using of a mobile-phone-based advanced symptom management system (ASyMS) to monitor and manage chemotherapyrelated toxicity. Euro J Oncol Nurs. 2008;12(4):380-6.

45. Maguire R, Ream E, Richardson A, Connaghan J, Johnston B, Kotronoulas G, et al. Development of a novel remote patient monitoring system: the advanced symptom management system for radiotherapy to improve the symptom experience of patients with lung cancer receiving radiotherapy. Cancer Nurs. 2015;38(2):E37-47.

46. McCarthy MC, Wakefield CE, DeGraves S, Bowden M, Eyles D, Williams LK. Feasibility of clinical psychosocial screening in pediatric oncology: implementing the PAT2.0. J Psychosoc Oncol. 2016;34(5):363-75.

47. Meldahl ML, Acaster S, Hayes RP. Exploration of oncologists' attitudes toward and perceived value of patient-reported outcomes. Qual Life Res. 2013;22(4):725-31.

48. Noble-Jones R, Thomas MJ, Bose P. The lymphoedema genitourinary cancer questionnaire in urology follow-up clinics. Int J Urol Nurs. 2019;13(1):5-12.

49. Osborne TR, Ramsenthaler C, de Wolf-Linder S, Schey SA, Siegert RJ, Edmonds PM, et al. Understanding what matters most to people with multiple myeloma: a qualitative study of views on quality of life. BMC Cancer. 2014;14(1):496.

50. Semple CJ, Lannon D, Qudairat E, McCaughan E, McCormac R. Development and evaluation of a holistic surgical head and neck cancer post-treatment follow-up clinic using touchscreen technology-feasibility study. Eur J Cancer Care. 2018;27(2):e12809.

51. Snyder CF, Blackford AL, Wolff AC, Carducci MA, Herman JM, Wu AW, et al. Feasibility and value of patientviewpoint: a web system for patient-reported outcomes assessment in clinical practice. Psychooncology. 2013;22(4):895-901.

52. Stover A, Irwin DE, Chen RC, Chera BS, Mayer DK, Muss HB, et al. Integrating patient-reported measures intoroutine cancer care: cancer patients' andclinicians' perceptions of acceptability and value. eGEMs. 2015;3(1):17.

53. Sundberg K, Eklöf AL, Blomberg K, Isaksson A-K, Wengström Y. Feasibility of an interactive ICT-platform for early assessment and management of patient-reported symptoms during radiotherapy for prostate cancer. Euro J Oncol Nurs. 2015;19(5):523-8.

54. Taylor S, Allsop MJ, Bekker HL, Bennett MI, Bewick BM. Identifying professionals' needs in integrating electronic pain monitoring in community palliative care services: an interview study. Palliat Med. 2017;31(7):661-70.

55. Thayssen S, Hansen DG, Søndergaard J, Høybye MT, Christensen PM Hansen HP. General practitioners' experience of using a questionnaire when assessing cancer patients' needs: a qualitative study. Fam Pract. 2016;34(1): $114-8$.

56. Thewes B, Davis E, Girgis A, Valery PC, Giam K, Hocking A, et al. Routine screening of indigenous cancer patients' unmet support needs: a qualitative study of patient and clinician attitudes. Int J Equity Health. 2016;15(1):90.

57. Velikova G, Brown JM, Smith AB, Selby PJ. Computer-based quality of life questionnaires may contribute to doctor-patient interactions in oncology. Br J Cancer. 2002;86(1):51-9.

58. Velikova G, Awad N, Coles-Gale R, Wright EP, Brown JM, Selby PJ. The clinical value of quality of life assessment in oncology practice-a qualitative study of patient and physician views. Psychooncology. 2008; 17(7):690-8.

59. Thorne S. Metasynthetic madness: what kind of monster have we created? Qual Health Res. 2017;27(1):3-12.

60. Bergdahl E. Is meta-synthesis turning rich descriptions into thin reductions? A criticism of meta-aggregation as a form of qualitative synthesis. Nurs Inq. 2019;26(1):e12273.

\section{Publisher's Note}

Springer Nature remains neutral with regard to jurisdictional claims in published maps and institutional affiliations.

\section{Ready to submit your research? Choose BMC and benefit from}

- fast, convenient online submission

- thorough peer review by experienced researchers in your field

- rapid publication on acceptance

- support for research data, including large and complex data types

- gold Open Access which fosters wider collaboration and increased citations

- maximum visibility for your research: over $100 \mathrm{M}$ website views per year

At BMC, research is always in progress.

Learn more biomedcentral.com/submissions 